\section{IN BRIEF}

\section{$\Rightarrow$ NEUROSCIENTIFIC COMMUNITY}

\section{Insight into citation bias}

Research suggests that papers by women tend to get cited less than those by men; however, the factors driving this gendered citation behaviour are not clear. Here, the authors used a probabilistic algorithm to assign genders to the first and last authors of 54,225 papers from five neuroscience journals since 1995 and found that papers with male first and last authors (man/man written papers) were cited $11.6 \%$ more than would be expected if randomly drawn references were cited, whereas woman/woman papers were cited $30.2 \%$ less than expected. The authors found that this imbalanced citation behaviour seems to be gradually increasing over time, and that it is mostly driven by the citation practice of man/man author teams.

ORIGINAL ARTICLE Dworkin, J. D. et al. The extent and drivers of gender imbalance in neuroscience reference lists. Nat. Neurosci. https://doi.org/10.1038/s41593-020-0658-y (2020)

\section{NAVIGATION}

\section{Putting bats on the cognitive map}

Whether bats navigate their large home range using a 'cognitive map' is debated. Toledo et al. and Harten et al. used GPS trackers to record flight trajectories of Egyptian fruit bats and provide evidence that these animals indeed use cognitive map representations. Toledo et al. tagged 172 bats for a total of 3,449 nights, resulting in 9,218 recorded trajectories. Of these, 397 were previously unrecorded shortcuts between two known locations. Moreover, bats translocated by Toledo et al. to the periphery of their main foraging area returned to their regular foraging area along new, straight trajectories. Various analyses suggested that the bats probably did not rely on other means of navigation such as random search, piloting, beaconing, path integration or following other bats. Harten et al. tagged wild bat pups with trackers before their first outdoor flights for the first few months of their lives. These young bats took shortcuts even in these early flights, suggesting that they develop cognitive maps of their home ranges during initial explorations.

ORIGINAL ARTICLES Toledo, S. et al. Cognitive map-based navigation in wild bats revealed by a new high-throughput tracking system. Science 369, 188-193 (2020)| Harten, L. et al. The ontogeny of a mammalian cognitive map in the real world. Science 369, 194-197 (2020)

\section{NEURODEGENERATIVE DISEASE}

\section{Hunting down vulnerabilities}

How the mutant huntingtin gene ( $m H T T$ ) causes neuronal death in Huntington disease (HD) is not known. Previous studies investigating the basis of its toxicity have not focused on striatal spiny projection neurons of the indirect pathway (iSPNs), which are the most vulnerable cell type in HD. Lee et al. used translating ribosome affinity purification and single-nucleus RNA sequencing (snRNA-seq) to find alterations in gene expression in different cell types in HD mouse models, and nRNA-seq to compare gene expression in different cell types in post-mortem samples from individuals with HD versus non-HD controls. Mitochondrial RNAs (mtRNAs) and RNAs involved in mitochondrial oxidative phosphorylation were upregulated and downregulated, respectively, in iSPNs from HD brain tissue, and immunoprecipitation revealed that $m t R N A$ s bind to the innate immune sensor protein kinase $R$. Thus, $\mathrm{mHTT}$ may induce $\mathrm{mtRNA}$ release in iSPNs, leading to innate immune activation.

ORIGINAL ARTICLE Lee, H. et al. Cell-type-specific transcriptomics reveals that mutant huntingtin leads to mitochondrial RNA release and neuronal innate immune activation. Neuron https://doi.org/10.1016/j.neuron.2020.06.021 (2020)

\title{
Misbalance in metabolism
}

Astrocytes provide metabolic support to neurons by metabolizing glucose to produce lactate, which neurons use for energy. owever, whether and how changes in astrocytic metabolism can affect neurons and behaviour is not clear. Now, Jimenez-Blasco et al. show in mice that activation of the mitochondrial membrane cannabinoid 1 receptor $\left(\mathrm{mtCB}_{1}\right)$ in astrocytes disrupts the metabolism of these cells and neurons, and induces deficits in social behaviour.

The authors identified $\mathrm{CB}_{1}$ in juxtaposition with astrocytic mitochondria. Treating cultured mouse astrocytes with a cellpermeable $\mathrm{CB}_{1}$ agonist (HU210 or THC), but not a cell-impermeable $\mathrm{CB}_{1}$ agonist, reduced mitochondrial complex I activity. This effect was restored in $\mathrm{CB}_{1}$-null astrocytes by re-expressing wild-type (WT) $\mathrm{CB}_{1}$, but not a form of $\mathrm{CB}_{1}$ excluded from mitochondria. Thus, $\mathrm{mtCB}_{1}$ activation reduces astrocyte metabolism through complex I.

In WT astrocytes and mouse brains, HU210 and THC reduced Ser173 phosphorylation of the complex I subunit NDUFS4 (NADH dehydrogenase (ubiquinone) iron-sulphur protein 4). By contrast, THC did not reduce NDUFS4 phosphorylation in $\mathrm{CB}_{1}$-null astrocytes. In addition, complex I function in astrocytes expressing a phosphomimetic form of NDUFS4 (NDUFS4-PM) was unaffected by $\mathrm{CB}_{1}$ agonism. The authors also showed that NDUFS4 Ser 173 phosphorylation is needed for complex I stability, thus indicating that $\mathrm{CB}_{1}$ activation destabilizes complex I by reducing NDUFS4 phosphorylation.

Previous research suggests that mitochondrial reactive oxygen species (mROS) generated by complex I are sensed by hypoxia-inducible factor 1 (HIF1), which in turn promotes glycolysis and lactate release. Here, THC reduced mROS production, HIF1 subunit levels and lactate release in WT, but not NDUFS4-PM-expressing or $\mathrm{CB}_{1}$-null, astrocytes. Moreover, neurons co-cultured with WT astrocytes that had previously been treated with THC or HU210 showed signs of bioenergetic stress that were ameliorated by lactate supplementation. Thus, astrocyte $\mathrm{CB}_{1}$ agonism disrupts the glycolytic support that astrocytes provide to neurons.

Next, the authors tested the effects of astrocyte $\mathrm{CB}_{1}$ activation on behaviour in two social interaction tasks. THC injection led to deficits in the two-chamber social interaction test in WT mice but not in mice lacking $\mathrm{CB}_{1}$ in astrocytes or in WT mice that had received an intracerebroventricular injection of lactate. Knockdown of monocarboxylate transporter 2 (which carries lactate into neurons) in the hippocampus and prefrontal cortex of mice also occluded the effects of THC treatment on social interactions, implying that the behavioural effects of astrocyte $\mathrm{CB}_{1}$ agonism are mediated by a reduction in the supply of lactate to neurons.

Together, these findings demonstrate that activation of astrocyte $\mathrm{mtCB}_{1}$ induces metabolic disruption in astrocytes and neurons, resulting in behavioural changes.

Natasha Bray

ORIGINAL ARTICLE Jimenez-Blasco, D. et al. Glucose metabolism links astroglial mitochondria to cannabinoid effects. Nature https://doi.org/ 10.1038/s41586-020-2470-y (2020) 